\section{Tuberkulose: Verbesserte Schnelldiagnostik bei Kindern}

Zar HJ et al. Tuberculosis Diagnosis in Children Using Xpert Ultra on Different Respiratory Specimens. Am J Respir Crit Care Med 2019; doi:10.1164/rccm.201904-07720C

Die Diagnose der Lungentuberkulose (PTB) bei Kindern ist aus mehreren Gründen schwierig: Die Erkrankung präsentiert sich mit unspezifischen klinischen oder radiologischen Symptomen, sie kann bakterienarm sein und mancherorts fehlen für die mikrobiologische Diagnose Kapazitäten, Erfahrung und Infrastruktur. Zur Verbesserung der Situation wurde die Genauigkeit und Leistungsfähigkeit des Xpert-Ultra-Tests untersucht.

Infektionen mit Mycobacterium tuberculosis (MTB) und möglicher gleichzeitiger Rifampicin-Resistenz (RIF) sollen mit dem Test Xpert MTB/RIF Ultra (Xpert) schneller und genauer diagnostiziert werden können. In seiner aktuellen Studie ging ein südafrikanisches Team der Frage nach, wie genau der Test ist und welche diagnostische Ausbeute sich mit ihm unter Verwendung wiederholt entnommener nasopharyngealer Aspirate (NPA) erzielen lässt. Außerdem untersuchten die Ärzte, ob die diagnostische Ausbeute sich steigern lässt, wenn NPA und induziertes Sputum (IS) kombiniert getestet werden. In die Studie wurden in Kapstadt, Südafrika, zwischen April 2012 und September 2017 konsekutiv Kinder im Alter von unter 15 Jahren mit einem PTB-Verdacht aufgenommen. Bedingungen waren ein häuslicher Tuberkulosekontakt innerhalb der letzten 6 Monate vor der Testung, Gewichtsverlust oder fehlende Gewichtszunahme während der letzten 3 Monate vor der Testung, ein positiver Tuberkulin-Hauttest oder ein Röntgenbefund mit PTB-Hinweisen. Die Kinder wurden in 3 diagnostische Kategorien eingeteilt, wobei dafür die XpertBefunde ausgeschlossen waren, um den Studieneinschluss nicht zu verzerren: bestätigte TB (bakteriologisch positiv für M. tuberculosis), unbestätigte TB (bakteriologisch negativ, klinische TB-Diagnose), TB unwahrscheinlich (bakteriologisch ne- gativ, keine klinische TB-Diagnose, keine TB-Therapie und dokumentierte Verbesserung zur Nachuntersuchung nach 3 Monaten).

\section{NPA und IS kombiniert verbessern Diagnose}

Insgesamt stand von 195 Kindern mit einem medianen Alter von 23,3 Monaten, von denen 16,4\% HIV infiziert waren, mindestens 1 Probe IS und NPA zur Verfügung. Bei 130 Kindern dieser Gruppe konnte ein zweites NPA getestet werden. 20,5\% der Kinder aus der Gesamtgruppe hatten eine bakteriologisch bestätigte TB, bei 53,3\% war sie unbestätigt und in $26,2 \%$ der Fälle nicht wahrscheinlich. Während insgesamt 57,2\% der Tuberkulin-Hauttests positiv waren, waren sie in der Gruppe mit bestätigter TB zu $91 \%$ positiv. Xpert war bei $13,3 \%$ der NPAs und bei $15,9 \%$ der IS-Proben positiv. Unter Verwendung eines NPA erreichte die Sensitivität des Schnelltests $46 \%$ und die Spezifität $98 \%$. Bezogen auf eine ISProbe betrug die Sensitivität 74,3\% und die Spezifität 96,9\%. Bei der Kombination eines NPA und eines IS erhöhte sich die Sensitivität des Xpert-Tests auf $80 \%$. Die Sensitivität von 2 NPAs allein erreichte $54,2 \%$, stieg in Kombination mit einem IS auf $87,5 \%$.

FAZIT

In dieser Studie konnte mit dem Schnelltest Xpert MTB/RIF Ultra die Mehrheit der durchschnittlich 2 Jahre alten Kinder mit bakteriologisch bestätigter Tuberkulose identifiziert werden, wenn nasopharyngeale Aspirate und induziertes Sputum kombiniert getestet wurden.

Matthias Manych, Berlin 